$\Rightarrow$ HYPERTENSION

\section{PIEZOs in}

\section{baroreceptor reflex}

The baroreceptor reflex enables the nervous system to sense and respond to changes in blood pressure (BP). These acute responses, in combination with long-term changes in heart rate $(\mathrm{HR})$ and $\mathrm{BP}$, maintain $\mathrm{BP}$ homeostasis.

Failure of the baroreflex response has adverse cardiorenal consequences; however, the identity of the mechanosensitive baroreceptors is unknown. Investigators now describe two mechanosensitive ion channels - PIEZO1 and PIEZO2 - that are required for baroreceptor activity. "Our study uncovers these two mechanosensitive proteins as key players in BP sensing," say Ardem Patapoutian and Wei-Zheng Zeng. "Mutations in PIEZO1 and PIEZO2 might therefore be risk factors for baroreflex dysfunction in humans."

The researchers used retrograde tracing of carotid sensory neurons combined with a sensitive in situ hybridization approach to identify Piezo1 and Piezo2 transcripts in baroreceptor cells. Given that constitutive deletion of Piezo1 and Piezo2 is lethal, the researchers generated mice in which these genes were specifically deleted in vagal neurons. Single Piezo1 and Piezo2 knockout mice showed no phenotype; however, double knockout of Piezo1 and Piezo2 abolished the baroreflex-induced reduction in HR in response to administration of the vasoconstrictor phenylephrine. Double knockout mice also exhibited labile hypertension and had greater BP variability than wild-type mice, consistent with findings in patients with baroreflex failure.

To evaluate the contribution of PIEZOpositive sensory neurons to $\mathrm{BP}$ regulation, the researchers manipulated the neural activity of PIEZO2-expressing baroreceptors using an optogenetics approach, showing that stimulation of PIEZO2-sensory neurons induces a baroreflex response with immediate decreases in both BP and HR. "We are interested in understanding how changes in the function of these proteins might affect BP regulation in humans," explains Patapoutian. "Moreover, our findings open new research avenues to study how neurons decode and process mechanical force information in the vascular system, and to identify new targets for treating hypertension and heart failure."

$\Rightarrow$ HYPERTENSION

\title{
ALMS1-NKCC2 interactions
}

Enhanced activity of the renal $\mathrm{Na}^{+} \mathrm{K}^{+} 2 \mathrm{Cl}$ transporter, NKCC2, has been linked to hypertension, but the mechanisms that regulate NKCC2 activity are largely unknown. New research now reports a role for Alström syndrome protein 1 (ALMS1) in mediating NKCC2 trafficking and activity. "Our study shows that ALMS1 dysfunction causes hypertension, decreases renal sodium excretion and increases NKCC2-mediated $\mathrm{NaCl}$ transport in the thick ascending limb (TAL)," explains Pablo Ortiz.

The carboxyl terminus of NKCC2 is important for its trafficking to and from the membrane. Using a targeted proteomics approach, Ortiz and colleagues identified ALMS1 as a protein that interacts with this region of NKCC2 and with other proteins involved in endocytosis, suggesting a role for ALMS1 in NKCC2 trafficking. To assess the functional relevance of AMLS1, the researchers generated ALMS1-knockout rats. These rats appeared largely normal by 12 weeks of age, but were hypertensive and exhibited increased sensitivity to high salt intake. Knockout rats were more sensitive to the diuretic and natriuretic effects of the
NKCC2 inhibitor bumetanide and had an impaired ability to respond to acute volume loading. Together these data showed that ALMS1-knockout rats exhibited higher $\mathrm{NaCl}$ reabsorption in the TAL than wild-type rats.

Finally, the researchers used a cell-surface biotinylation assay to show that deletion or silencing of ALMS1 decreased NKCC2 endocytosis, causing it to accumulate at the cell surface. "These findings explain how defective ALMS1 induces NKCC2-mediated $\mathrm{NaCl}$ transport in the TAL, and provides an explanation for some of the syndromic characteristics of Alström syndrome, which is caused by loss-of-function mutations in ALMS1," says Ortiz. "Moreover, singlenucleotide polymorphisms in ALMS1 are associated with renal dysfunction, obesity and metabolic syndrome. It would be interesting to now look at whether this gene is associated with hypertension in the general population."

Susan J. Allison

ORIGINAL ARTICLE Jaykumar, A. B. et al. Role of Alström syndrome 1 in the regulation of blood pressure and renal function. JCl Insight https://doi.org/10.1172/jci.insight.95076 (2018)

\section{PRE-ECLAMPSIA}

\section{A novel RNA interference approach to targeting sFLT1 in pre-eclampsia}

Abnormally high levels of soluble FLT1 (sFLT1; also known as sVEGFR1) have a key role in the pathogenesis of pre-eclampsia. Now, Ananth Karumanchi and colleagues have developed an RNA interference (RNAi) approach to modulate sFLT1 expression that ameliorates pre-eclampsia in animal models.

"Our desire was to develop a simple and cost-effective pre-eclampsia therapeutic using RNAi to limit excess placental expression of sFLT1 proteins," explains Karumanchi. "Placental sFLT1 expression is dominated by three truncated mRNA isoforms that are generated by polyadenylation within Flt 1 introns 13 and 14. Targeting these intronic regions using RNAi enables silencing of the abnormally expressed truncated mRNA variants that encode sFLT1 proteins without interfering with the expression of full-length FLT1."

Using a systematic screening process, the researchers identified two hydrophobically modified asymmetric small interfering RNAs (hsiRNAs) that selectively silenced sFLT1
mRNAs in cultured human cytotrophoblasts. Following intravenous administration, chemically stabilized, cholesterol-conjugated versions of these hsiRNAs accumulated in the placentas of pregnant mice and reduced circulating sFLT1 levels with no adverse effects on the animals or their pups.

Similarly, injection of the hsiRNAs substantially reduced the levels of circulating sFLT1 proteins in a baboon model of pre-eclampsia. The treated animals showed reductions in blood pressure and proteinuria but no significant difference in birth weights compared with controls.

"I believe that our study demonstrates that we can target the placenta safely," comments Karumanchi. "Therefore, our approach opens the door for a variety of other placental targets for pre-eclampsia and other related disorders."

Ellen F. Carney

ORIGINAL ARTICLE Turanov, A. A. et al. RNAi modulation of placental sFLT1 for the treatment of preeclampsia. Nat. Biotechnol.https://doi.org/10.1038/nbt.4297 (2018) 